\title{
Le
}

SECCIÓN: Educación y espacio
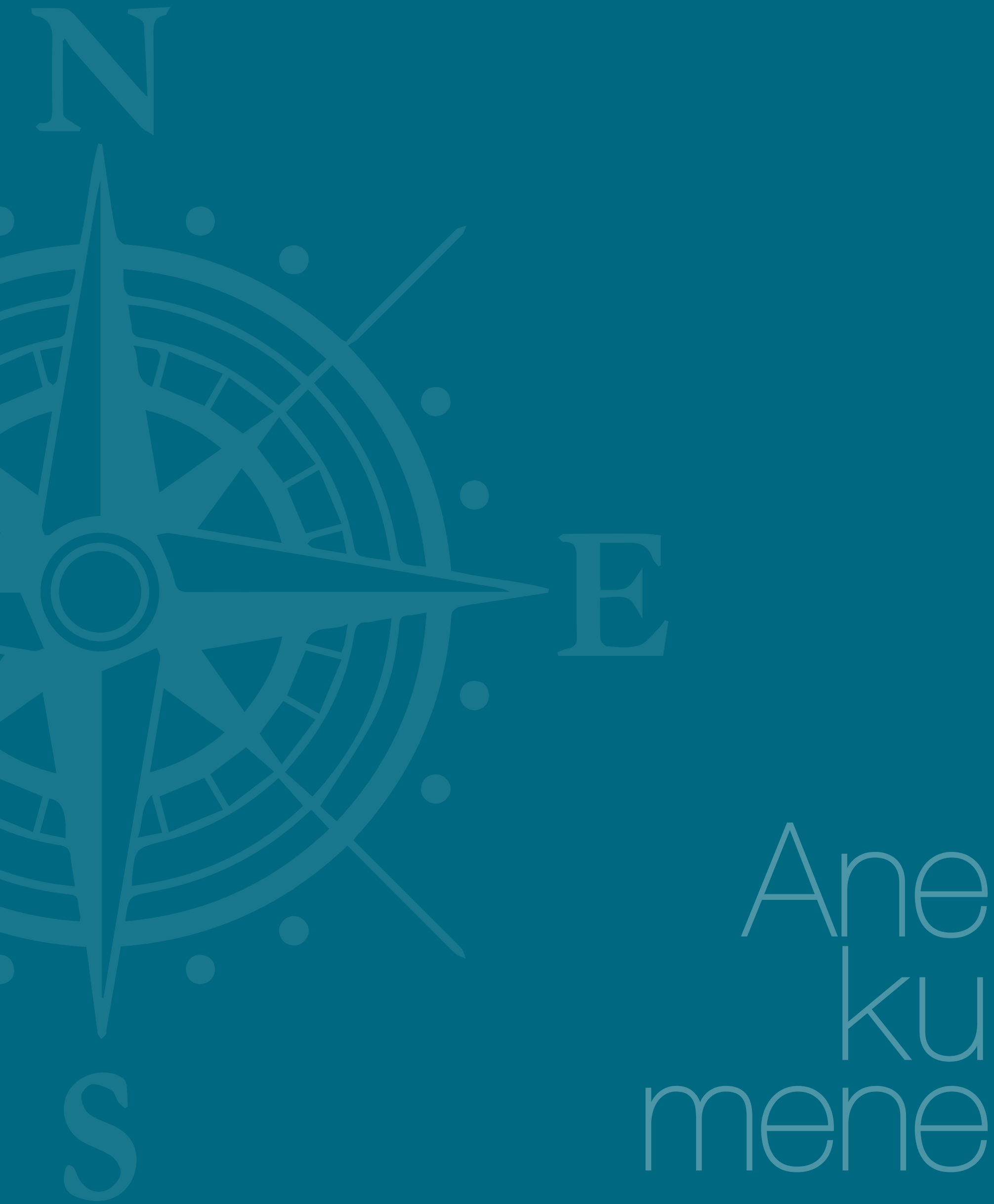


\section{La construcción del conocimiento en el aula: la tarea esencial de la enseñanza geográfica}

\section{The Construction of Knowledge in the Classroom: the Essential Task of Geographical Education}

\section{A construção de conhecimentos na sala de aula: a tarefa esencial da educação geográfica}

José Armando Santiago Rivera*

\begin{abstract}
Resumen
El propósito es analizar las orientaciones geográficas y pedagógicas para construir el conocimiento en la práctica escolar cotidiana de la enseñanza de la geografía. Se asume que la exigencia de su innovación formativa obedece a los desafíos originados por las circunstancias de la época contemporánea, y los cambios paradigmáticos y epistemológicos, como el incremento de las necesidades de la sociedad. En efecto, se examinan los fundamentos teóricos y metodológicos de la ciencia geográfica, la pedagogía y la didáctica que se han puesto en práctica para guiar los procesos de enseñanza y de aprendizaje, en especial la vigencia de los conocimientos y prácticas decimonónicas notablemente contradictorias con las realidades del actual momento histórico impregnado del nuevo orden económico mundial. Eso implica considerar la importancia del dictado, el dibujo, la copia y el calcado como las formas de aprender nociones y conceptos geográficos; la fijación en la mente de los estudiantes de los contenidos programáticos; la relevancia de elaborar el conocimiento desde las ideas previas y construir el conocimiento a partir de la experiencia ciudadana de los estudiantes, dada su participación
\end{abstract}

activa y protagónica como habitantes de una comunidad. Por tanto, estos aspectos se explicarán en la perspectiva de la exclusividad de la ciencia positiva y los planteamientos paradigmáticos y epistemológicos de la ciencia cualitativa. Al respecto, metodológicamente, se realizó una revisión de bibliografía que sirvió para desarrollar una reflexión analítica sobre el panorama histórico contemporáneo, la transmisión del contenido programático en el aula, la crítica a la transmisión del contenido programático y opciones factibles de contribuir a la innovación desde la construcción del conocimiento en la enseñanza de la geografía. Se concluye resaltando los retos y los desafíos que enfrenta este campo del conocimiento científico y pedagógico en el mundo contemporáneo ante tanto el incremento de las dificultades ambientales, geográficas y sociales, como la exigencia de contribuir a sensibilizar y alfabetizar los ciudadanos sobre el deterioro ecológico, el uso del territorio y la organización de su espacio geográfico.

\section{Palabras claves}

construcción del conocimiento; enseñanza geográfica. 


\section{Abstract}

The purpose is to analyze the geographic and pedagogical orientations to construct the knowledge in the daily school practice of the teaching of the geography. It is assumed that the demand for its formative innovation obeys the challenges originated by the circumstances of the contemporary era, the paradigmatic and epistemological changes, such as increasing the needs of society. In fact, we examine the theoretical and methodological foundations of geographical science, pedagogy and didactics that have been put in place to guide teaching and learning processes; in particular, the validity of nineteenth-century knowledge and practices that are remarkably contradictory to the realities of the current historical moment imbued with the new world economic order. This implies considering the importance of dictation, drawing, copying and footing as ways of learning notions and geographical concepts; the fixing in the students' minds of the programmatic contents; the relevance of developing knowledge from previous ideas and building knowledge from the citizens' experience of the students, given their active and protagonist participation as inhabitants of a community.
Therefore, these aspects will be explained in the perspective of the exclusivity of positive science and the paradigmatic and epistemological approaches of qualitative science. In this regard, methodologically, a bibliographical review was carried out that served to develop an analytical reflection on the contemporary historical panorama, the transmission of the programmatic content in the classroom, the criticism to the transmission of the programmatic content and feasible options to contribute to the innovation since the construction of knowledge in the teaching of geography. It concludes by highlighting the challenges and challenges facing this field of scientific and pedagogical knowledge in the contemporary world in the face of increasing environmental, geographic and social difficulties, as well as the need to contribute to sensitize and educate citizens about ecological deterioration, the use of the territory and the organization of its geographical space.

\section{Keywords}

knowledge construction; geographical teaching.

\section{Resumo}

O objetivo é analisar as orientações geográficas e pedagógicas para construir o conhecimento na prática escolar diária do ensino da geografia. Assume-se que a demanda por sua inovação formativa obedece aos desafios originados pelas circunstâncias da era contemporânea, as mudanças paradigmáticas e epistemológicas, como o aumento das necessidades da sociedade. De fato, examinamos os fundamentos teóricos e metodológicos da ciência geográfica, pedagogia e didática que foram implementados para orientar os processos de ensino e aprendizagem; em particular, a validade do conhecimento e das práticas do século XIX que são incrivelmente contraditórias às realidades do momento histórico atual imbuído da nova ordem econômica mundial. Isso implica considerar a importância do ditado, desenho, cópia e suporte como formas de aprender noções e conceitos geográficos; a fixação na mente dos estudantes dos conteúdos programáticos; a relevância do desenvolvimento de conhecimento de idéias anteriores e a construção de conhecimento a partir da experiência dos cidadãos dos alunos, dada a sua participação ativa e protagonista como habitantes de uma comunidade. Portanto, esses aspectos serão explicados na perspectiva da exclusividade da ciência positiva e das abordagens paradigmáticas e epistemológicas da ciência qualitativa. A este respeito, metodologicamente, realizou-se uma revisão bibliográfica que serviu para desenvolver uma reflexão analítica sobre o panorama histórico contemporâneo, a transmissão do conteúdo programático na sala de aula, a crítica à transmissão do conteúdo programático e opções viáveis para contribuir com a inovação desde a construção do conhecimento no ensino da geografia. Conclui destacando os desafios e os desafios que enfrenta este campo do conhecimento científico e pedagógico no mundo contemporâneo, face às crescentes dificuldades ambientais, geográficas e sociais, bem como a necessidade de contribuir para sensibilizar e educar os cidadãos sobre a deterioração ecológica, o uso do território e a organização do seu espaço geográfico.

\section{Palavras-chave}

construção do conhecimento; ensino geográfico. 


\section{Introducción}

En el mundo contemporáneo, es claro que la atención sobre la educación es evidencia de la exigencia social de formar a los ciudadanos con la capacidad de explicar crítica y constructivamente la realidad sociohistórica de su época. Como muestra de esa finalidad, en el debate teórico se promueve el modelo educativo humanístico, aunque en la práctica escolar cotidiana todavía están vigentes las orientaciones formativas establecidas en el siglo XIX.

Esta situación revela una preocupante discrepancia porque el acto educante debería ser coherente con las condiciones contemporáneas científico-tecnológicas, donde la asombrosa innovación que le caracteriza aporta conocimientos y prácticas novedosas para examinar las situaciones ambientales, geográficas y sociales desde ópticas analíticas e interpretativas de acento integral, ecológico, sistémico, vivencial y holística.

Lo alarmante es que en la enseñanza de la geografía predomina la transmisión de contenidos programáticos referidos a los aspectos físico-naturales de la superficie terrestre, en condición de parcelas cognitivas concebidas como fragmentos de la naturaleza. Esta labor desvía la atención sobre las problemáticas derivadas de la ocupación del territorio y la organización del espacio geográfico.

Esta problemática determinó, metodológicamente, la realización de la consulta bibliográfica para estructurar una reflexión sobre la realidad geográfica del mundo globalizado, los sucesos de la práctica pedagógica de la geografía escolar, y explicar los argumentos geográficos pedagógicos y didácticos que pueden estimular la elaboración del conocimiento desde la acción investigativa de lo real.

La justificación de estos razonamientos obedece a la necesidad de la actividad indagadora de las temáticas y las problemáticas que afectan a la sociedad, especialmente en su entorno inmediato. De allí el interés por considerar los avances epistémicos de la revolución paradigmática como base de iniciativas conducentes a mejorar la calidad formativa de la enseñanza geográfica.

Hoy día es imprescindible que este campo del conocimiento científico y pedagógico sea coherente con los retos y desafíos enfrentados por América Latina y el Caribe. Eso implica promover la enseñanza de la geografía desde una orientación más humana y social, con fines de alfabetizar las colectividades en forma acorde a los cambios geohistóricos del inicio del nuevo milenio.

\section{La innovación en la explicación de la realidad geográfica}

Precisamente ya hacia el final del siglo XX, la extraordinaria divulgación de noticias, informaciones y conocimientos, referenciados como la "Explosión de la información" y "la sociedad del conocimiento", reveló la compleja realidad geográfica en su multiplicidad y diversidad de temáticas y problemáticas, aunque fue común apreciar no solo la exclusividad de la ciencia positiva, sino también la presencia de otras opciones explicativas de los acontecimientos geográficos.

En ese sentido, en la perspectiva de Claval (1992), la ciencia geográfica estudiaba temáticas como el subdesarrollo; la dependencia; la ruptura del equilibrio ecológico; la contaminación ambiental; el crecimiento desordenado de los centros urbanos; la dinámica geopolítica; la crisis del sistema capitalista mundial; la economía globalizada y su efecto en la desigualdad social. Otros temas también fueron la crisis del modelo económico capitalista; la dinámica geopolítica hegemónica; el sentido abierto de las fronteras políticas; la intensa movilidad demográfica surnorte; las diferencias étnicas, raciales y la exclusión social; y la ocurrencia cotidiana de diversos conflictos, infortunios, catástrofes y adversidades de notable efecto social.

Estas problemáticas estimularon a la docencia y la investigación científica de la geografía a aplicar modelos matemáticos y estadísticos, la percepción espacial, la interpretación de los cambios históricos del espacio, la geografía fenomenológica, los sistemas de información geográfica, entre otras alternativas explicativas, en la dirección del análisis renovado y crítico de las causalidades geográficas.

Sin embargo, a finales del siglo XX, fue necesario reconocer los aportes de la ciencia cualitativa para interpretar hermenéuticamente los hechos desde la subjetividad de sus actores y descifrar los sucesos a partir del sentido común, la intuición y la experiencia cotidiana de los ciudadanos. El logro de este aporte fue superar la contemplación objetiva de los objetos de estudio para exigir la intervención vivencial, dialéctica y crítica de sus actores protagónicos.

El cambio significó, en la reflexión de González y Ojeda (2005), que el investigador se debió involucrar activamente en el acto interpretativo de la realidad desde una postura participativa; por ejemplo, debía asumir los imaginarios de los ciudadanos para conocer los efectos del momento histórico en el lugar y analizar las penurias sociales en la perspectiva geográfica, ideológica y política.

De esta forma, la ciencia geográfica, en la opinión de Hernández (2011), colocó en tela de juicio la exclusividad del positivismo, apoyado en la dirección epistémica del método hipotético-deductivo valorada como 
la opción para conocer en forma objetiva, rigurosa y estricta. Por cierto, esta visión científica ya apuntalaba desde el siglo XIX la formación educativa de la geografía escolar.

La ciencia positiva, según González (2009), representó “La pretensión de estudiar la realidad parte por parte que significa poner en práctica el esquema simplificador y reduccionista en la construcción del conocimiento, pues un conocimiento que se fracciona produce inevitablemente un saber mutilado e incompleto" (p. 64).

Su aplicación en la enseñanza geográfica durante tres siglos ha sido objeto de la crítica de Pipkin, Varela y Zenobi (2001), para quienes el cuestionamiento obedece a la vigencia de la transmisión de contenidos programáticos y el descarte del estudio de los problemas sociales, pues "Los alumnos los conocen a partir de los medios de comunicación social pero no siempre pueden interpretarlos, contextualizarlos, ni encuentran relaciones entre ellos y lo que aprenden en la escuela" (p. 4).

Por tanto, en la actualidad en la práctica escolar cotidiana de la enseñanza de la geografía se aprecia, por un lado, la validez de la orientación positivista y, por el otro, la versión de la ciencia de acento cualitativo. El resultado es que, mientras permanecen casi intactos los fundamentos decimonónicos en el aula de clase, lo cualitativo es solamente planteamiento teórico, para innovar lo pedagógico y lo didáctico.

Esta situación ha colocado a la geografía escolar en una situación discrepante de la complejidad del mundo contemporáneo, por su acento en la reproducción libresca y la memorización de los conceptos. En efecto, su marcada distancia con las enrevesadas circunstancias comunitarias, y la renovación paradigmática y epistemológica derivan en una labor formativa impropia, inadecuada y obsoleta para que la sociedad comprenda las actuales condiciones geohistóricas.

De allí surge la obligación de revisar los fundamentos de la geografía escolar al enseñar el conocimiento geográfico. Indiscutiblemente, el reto es reconciliar esta acción educativa con el panorama sociohistórico y las formas de elaborar el conocimiento desde epistemes constructivas y críticas, con el propósito de redireccionar la orientación pedagógica acorde con la época globalizada, la complejidad vivida y las necesidades de la sociedad.

Así, innovar la enseñanza de la geografía en su trabajo escolar cotidiano obedece a la necesidad tanto de dar respuesta a los retos y los desafíos que enfrenta este campo del conocimiento científico y pedagógico en el mundo contemporáneo, como, en especial, de mejorar la calidad formativa de la educación geográfica para que sea pertinente con la transformación de la complicada realidad ecológica, ambiental y geográfica de América Latina y el Caribe.

\section{La situación de la práctica pedagógica de la geografía escolar}

La imperiosa exigencia de entender el complejo, contradictorio e incierto desenvolvimiento del actual momento histórico obedece a los preocupantes "desastres naturales", cuya eventualidad cotidiana permite asignar a la época el calificativo de difícil, caótica y adversa, ante el suceder de habituales fatalidades, adversidades, calamidades y catástrofes que afectan a la población en las diferentes regiones del planeta.

En ese contexto, también es impresionante el ritmo de la invención, la creatividad y la originalidad que se muestra en los diferentes órdenes del sistema integral de la sociedad, en especial en la ciencia y la tecnología, aunque es sorprendente e incomprensible apreciar sus repercusiones poco efectivas y escasamente contundentes en el mejoramiento de la calidad de vida del conglomerado demográfico mundial.

Esta circunstancia ha sido motivo para estimular la innovación del modelo educativo hacia la formación de ciudadanos con conciencia cuestionadora sobre las dificultades confrontadas, pero, del mismo modo, estimar iniciativas de cambio y transformación que superen las debilidades, luego convertidas en significativas oportunidades, es decir, educar con el propósito de contribuir con opciones que mejoren las condiciones de vida social.

Por cierto, desde mediados del siglo XX, las reformas curriculares han previsto teóricamente apoyar el viraje formativo para educar de manera pertinente con las realidades del momento sociohistórico. En esta diligencia, un tema de interés ha sido revisar la práctica escolar cotidiana de la enseñanza geográfica, pues allí, en la opinión de Moreno, Rodríguez y Sánchez (2007),

[...] los programas y propuestas curriculares aunque cambian de contenido no se han transformado en lo esencial. Todavía se sigue enseñando una geografía cosificada, carente de vida, recortada en diversos planos [...], desde el punto de vista ideológico continúa con su pretensión de neutralidad frente al mundo de la vida, objetualizando al espacio como a los mismos sujetos, sin llegar a comprender la fundamental interacción entre individuos y entornos para la construcción significativa del mundo geográfico. (p. 91).

Lo enunciado significa la disonancia entre lo teórico y la práctica. Mientras el currículo se sostiene con fundamentos teóricos y metodológicos renovados, la actividad escolar cotidiana está sumergida en el tradicionalismo. Así mismo, aunque la geografía como disciplina científica avanza vertiginosamente en sus conocimientos y prácticas, se enseña con la orientación descriptiva. 
Lo llamativo es que en las reformas curriculares se inquietan por una orientación humanística, con una labor orientada a fortalecer los valores, como la responsabilidad y el compromiso social, en cambio en la actividad del aula la acción pedagógica se aferra a la actividad transmisiva, para poco aplicar los aportes pedagógicos y didácticos de acento innovador y novedoso.

Al realizar una reflexión sobre este suceso, resalta el hecho de que el acento tradicionalista está atrapado en los aspectos teóricos enumerativo, naturalista y determinista de la ciencia geográfica. En lo pedagógico y lo didáctico, hay un centramiento en los contenidos programáticos, facilitados desde la didáctica decimonónica para educar ciudadanos pasivos, acríticos e indiferentes a los hechos de la época.

Esta labor formativa ha sido objeto del cuestionamiento, desde finales de la década de los ochenta del siglo XX, ante su permanencia bajo el calificativo de extraviada y equivocada. Por ejemplo, en ese momento, al estudiar esta situación, Lacueva y Manterola (1989) criticaron la presencia de actividades didácticas como: copiar el contenido del libro, copiar el esquema del pizarrón, hacer ejercicios pautados en el libro, copiar lo dictado, escuchar la explicación del docente, entre otras.

Ante la necesidad de renovar esta enseñanza, se ha manifestado la estética del falso activismo pedagógico, donde, para citar un caso, el dictado es sustituido por la copia del contenido directamente del libro y el docente supervisa esa labor. Lo cierto es que persisten las actividades pretéritas reproductivas maquilladas, de tal manera que no se altera la formalidad enraizada en el aula desde el siglo XIX.

Esta geografía escolar, en el punto de vista de Rodríguez de Moreno (2000), implica reconocer "[...] que en la práctica pedagógica existen inconsistencias y contradicciones, entre el discurso académico, la enseñanza y la práctica de los docentes lo que se refleja en el panorama escolar, a pesar de las reformas educativas” (p. 11).

Esta realidad demuestra la complejidad de la concepción educativa, pues resulta un complicado contratiempo cuyo propósito es intelectualizar a los ciudadanos, con la perspectiva geográfica descriptiva y la didáctica transmisiva como orientaciones educativas fundamentales. En efecto, se da origen a una deficitaria formación que, en palabras de Delgado (2003), deriva en una débil actividad pedagógica, ya que

Las clases de geografía no convencen, pues con honrosas excepciones sólo se enseña una geografía corográfica en la que se intenta dar una información acerca de unos determinados países. Y por desgracia esas descripciones a base de mapas, cuadros sinópticos y apretados resúmenes, son menos interesantes que las que ofrecen los llamados mass-media, y por supuesto más pobre. (p. 2).
Esta realidad se erige como un obstáculo epistémico por el hecho de simplemente facilitar la acción didáctica hacia el contenido programático para obviar lo significativo de la explicación y la transferencia del concepto en la explicación de la realidad geográfica. Ante la prioridad de transmitir la noción o el concepto, este acto pedagógico resulta ingenuo, inocente, poco honesto e irresponsable.

Es indiscutible que se trata de una extraordinaria debilidad y permanente amenaza, porque utiliza con frecuencia técnicas verbales con fines de mantener la disciplina, el orden y la pasividad estudiantil, y no para facilitar propuestas innovadoras que asuman la explicación analítico-crítica de los problemas de su comunidad; es el uso arbitrario de experiencias más relacionadas con transmitir conceptos que estimular la elaboración del conocimiento.

Esta geografía escolar es calificada por Gurevich (1994) de la manera siguiente: "La geografía escolarizada es una versión lavada y descolorida de la realidad contemporánea. Ella describe trazos del planeta relatando sus características como si fueran postales congeladas" (p. 64). Por tanto, es impropia y distante de la explicación de la crisis ecológica, el hacinamiento urbano, la contaminación ambiental, entre otros aspectos.

En consecuencia, es apremiante que la enseñanza geográfica rompa con las ataduras establecidas en el siglo XIX. Un paso decisivo es abordar lo enrevesado del entorno inmediato y asumir el acento científico del acto de enseñar y aprender, desde la perspectiva de la ciencia cualitativa, fundada en lo fenomenológico, la etnografía, las historias de vida y la investigación acción, para evitar el recetario positivista-conductista. Eso obedece, según Rodríguez (2008), a lo siguiente:

[...] la geografía escolar ya no puede competir en calidad con la información transmitida por los medios de comunicación, a lo que habría que apostillar que ni debe hacerlo, pero si le compete ahora integrar esa información geográfica "popular" como objeto de análisis crítico para formar el futuro ciudadano a enfrentarse con el sistema de conformación de opiniones públicas que constituyen los medios de comunicación. (p. 26).

Entonces, una opción alterna puede ser que la innovación de la enseñanza de la geografía debería abordar analíticamente las condiciones geográficas del lugar, desde la capacidad interpretativa, crítica y creativa de los sucesos comunitarios. Allí será esencial y básico considerar la causalidad de los procesos geohistóricos del contexto inmediato urbanizado por los grupos humanos, al aprovechar las condiciones del territorio, percibidas desde los puntos de vista de sus habitantes.

Eso supone para la práctica pedagógica de la geografía escolar comenzar por reivindicar el sentido y el significado empírico forjado por los habitantes de la comunidad ante su condición de actores de los acontecimientos cotidianos. No es el hecho solamente de vivirlos, sino también del efecto en su subjetividad y en su desempeño como persona. 
Por tanto, es el ciudadano involucrado en su realidad geográfica, cuya experiencia es de fundamental importancia para mejorar la calidad formativa del acto educante.

\section{Para innovar la geografía escolar con la elaboración del conocimiento}

La aspiración de un cambio contundente en la enseñanza geográfica supone entender que la geografía científica y su enseñanza se desenvuelven en el marco de una época complicada colmada de cambios significativos, pero también de adversidades. Este desfase deja mal parados a los asombrosos avances de la ciencia y la tecnología, ante el incremento del hambre, el desempleo, la exclusión, el racismo y, en forma generalizada, la pobreza.

Este suceso no debe ni se puede tratar con la superficialidad transmisiva, sino con un modelo educativo que promueva procesos de enseñanza y aprendizaje desenvueltos en el escenario comunitario con responsabilidad y compromiso social. Eso implica activar la participación y el protagonismo social, porque la realidad es vivencia en acelerada inestabilidad, desequilibrio y versatilidad.

Ese salto epistémico hacia la elaboración del conocimiento debe considerar la perspectiva hermenéutica para descifrar los hechos vividos, en forma dialéctica y cuestionadora, analizados en su propia localidad. Esta iniciativa debe asumir la explicación en el marco del sentido acelerado del tiempo, la incertidumbre, la paradoja y el contrasentido. Lo enunciado supone lo siguiente:

\section{a) Entender el objeto de estudio en el complicado mundo contemporáneo}

Comprender el desenvolvimiento de la realidad estudiada exige realizar el análisis en el ámbito sociohistórico del mundo globalizado, pues se pretende educar al ciudadano con una formación que vigorice la conciencia crítica, desarrolle mecanismos formativos de activa y cuestionadora reflexión de su propia práctica y contribuya a fortalecer la vida democrática.

Es construir otros conceptos, desde el análisis dialéctico, controversial y metódico para descifrar la complejidad vivida. Eso se justifica en la perspectiva de González (2009), quien afirmó: “Lo cierto es que vivimos en un mundo socialmente complejo, en contextos complejos. [...] Tenemos que lograr que los actores educativos palpen, vivan y comprendan la complejidad" (p. 64).

Si se trata de entender lo enrevesado del momento y del lugar, la enseñanza de la geografía debe ejercitar de manera habitual la reflexión analítico-critica, la creatividad, la originalidad y la motivación al cambio innovador. En eso, la prioridad debe ser estimular los razonamientos que faciliten interpretar lo real, desde las visiones personales fundadas en la convivencia explicativa del territorio y desdibujado a partir de hallazgos investigativos.

\section{b) Asumir la innovación paradigmática y epistemológica}

Si la realidad se comporta complicada, lamentablemente con el positivismo se dificulta su comprensión, de allí que sea razonable, en el propósito de alfabetizar geográficamente a los ciudadanos, enseñar geografía con el desenvolvimiento de procesos investigativos desde la orientación cualitativa de la ciencia. Al respecto, es necesario incentivar los razonamientos analíticos, los cuales son ajustados a los fundamentos teóricos y metodológicos de la investigación participativa, entre otras epistemes, de esa naturaleza científica.

Es imprescindible vincular la acción pedagógica con las necesidades de la sociedad, y opinar analíticamente sobre la época en sus realizaciones y contratiempos. Así, la finalidad será enseñar geografía con sentido humano y social en sus propias localidades. En la perspectiva holística propuesta por Caldera (2008), significa considerar la posibilidad epistémica de la ciencia cualitativa para asumir la realidad, desde los puntos de vista de sus actores.

\section{c) Plantear una acción didáctica comprometida con el cambio social}

La interpretación de la realidad geográfica inmediata requiere de una acción didáctica innovadora que involucre a quien aprende en la realidad vivida. Es enseñar desde las ideas previas, el bagaje empírico y las interpretaciones personales sobre la realidad geográfica comunitaria, como base de los procesos pedagógicos y didácticos estructurados con actividades desencadenables hacia la realimentación en lo conceptual, lo metodológico y lo actitudinal.

En esta intervención geográfica se debe valorizar el desempeño ciudadano en sus vivencias y sus representaciones simbólicas sobre el mundo, la realidad y la vida, es decir, lo que manifiesta en sus puntos de vista sobre los actos comunitarios de su lugar. Es la didáctica de un modelo educativo que Giroux (2009) define como la educación contextualizada:

Es aquella que utiliza el entorno como recurso pedagógico. Una educación contextualizada será aquella que motive las relaciones del conocimiento con el contexto real del individuo y que lleve al conocimiento más allá, examinando las situaciones de otros contextos, analizando sus contradicciones y encuentros.

Desde este planteamiento, la enseñanza de la geografía va al escenario de los sucesos de la vida cotidiana para educar en la realidad misma, 
y donde los aprendizajes podrán renovarse y transformarse desde el fomento de la reflexión analítico-crítica desenvuelta en lo inmediato. De esta forma, elaborar el conocimiento geográfico será intervenir didáctica y dialécticamente la comunidad para entender y transformar su realidad vivida.

\section{d) Indagar el escenario comunitario con la aplicación del diagnóstico geográfico}

Ante la necesidad de una formación desde la interpretación geográfica del lugar, el punto de partida es reconocer el ámbito geográfico comunitario en su cotidianidad. El propósito es tanto explorar con el diagnóstico científico y pedagógico e identificar temáticas de interés social, como integrar lo descrito con los contenidos programáticos y organizar el proceso formativo con el acento investigativo.

Al analizar este planteamiento, desde la orientación didáctica del constructivismo, Carretero (2009) opinó lo siguiente: "el estudiante debe ser animado a conducir su propio aprendizaje, de esta manera la experiencia adquirida facilitará el aprendizaje al ocuparse más de los procedimientos y competencia que de los conocimientos estrictos" (p. 6).

Desde esta acción formativa, lo conveniente y apropiado de la integración entre vivencia, experiencia, sentido común e intuición, y la temática seleccionada será echar las bases del proceso pedagógico y didáctico donde se direccionará la relación entre el contenido geográfico con el objeto de estudio seleccionado. Es comenzar a formar al ciudadano en la lectura interpretativa de su propia realidad geográfica.

\section{e) Promover la acción hermenéutica de la realidad geográfica}

El salto epistémico es avanzar desde el ciudadano espectador que contempla ingenua, neutral y apolíticamente su entorno inmediato, al ciudadano autónomo y democrático. Es ejercitar la integración entre el contenido con la experiencia y la elaboración de puntos de vista; es una opción factible de establecer el puente entre la labor didáctica del aula con la comunidad a partir de la problematización de aspectos que afectan a las sociedades.

Se trata de una direccionalidad planteada desde la formulación de interrogantes originados en la lectura y la interpretación del contenido programático. En ese sentido, esta opción didáctica asume, desde la perspectiva de Díaz (1996), la necesidad de que los estudiantes pongan en práctica sus criterios personales y los fundamentos adquiridos sobre el tema o el problema geográfico para guiar su intervención en la búsqueda de una nueva explicación y otro conocimiento.

Desde los aspectos descritos, la enseñanza de la geografía podrá contribuir a innovar su tarea formativa. Así, conocer será una injerencia sistemática fundada en la experiencia ciudadana, la convivencia democrática y la acción metódica para obtener la información y su procesamiento hacia la elaboración de un nuevo conocimiento. Uno de los logros formativos serán los nuevos puntos de vista afianzados en lo real y coherentes con su análisis lógico de lo que allí sucede.

Lo enunciando representa, en palabras de Díaz y Hernández (2002), la oportunidad para elaborar el conocimiento desde un procesamiento integrador del contenido con la realidad en la promoción de los aprendizajes significativos, desde la aplicación de estrategias didácticas afincadas en la investigación. De esta forma, la enseñanza geográfica contribuirá a mejorar las necesidades sociales y entender los retos que enfrenta en la compleja situación del mundo actual.

Las circunstancias derivadas del aprovechamiento de las potencialidades del territorio latinoamericano y caribeño exigen estudiar la forma cotidiana del proceso formativo del aula. En principio, se impone examinar los fundamentos teóricos y metodológicos aplicados en la enseñanza geográfica para orientar los procesos formativos. De allí emergerá el requerimiento de revisar qué, cómo, por qué y para qué se ha enseñado la geografía en América Latina y el Caribe.

En consecuencia, se revelará la concepción geográfica distorsionadora de la forma como se aprovechan sus recursos naturales, al recurrir a una geografía descriptiva que simplemente aprecia la fisionomía de la superficie terrestre. Mientras, en lo pedagógico y lo didáctico ejercitan la reproducción conceptual y, con eso, desnaturalizan la comprensión de la organización espacial colonial y del nuevo orden económico mundial.

En efecto, desde la perspectiva tradicional, la enseñanza de la geografía se manifiesta como instrumento manipulador y alienante para desnaturalizar y obnubilar a los ciudadanos de la perversa acción destructiva de sus territorios. Este desfase conduce a informar a los ciudadanos desde la enseñanza geográfica convertida en un verdadero obstáculo epistémico que refuerza el desgano, la apatía y la indiferencia para descalificar su dignificante tarea educativa.

Ya es hora de que su innovación pedagógica y didáctica apunte hacia la formación del ciudadano culto, autónomo, cuestionador y democrático, por eso es razonable aprovechar la importancia adquirida por la ciencia cualitativa como oportunidad para la enseñanza de la geografía de ajustarse en conocimientos y estrategias innovadoras acordes con la renovación de los procesos de enseñanza y de aprendizaje hacia la construcción del conocimiento, con sentido humano y social.

El desafío es entender que la democracia debe ser fortalecida desde el desarrollo del pensamiento crítico, cooperador, reflexivo y liberador, el incentivo de una remozada didáctica fundada en la investigación flexible, 
comprometida, responsable, fraterna y solidaria. Ese es el desafío de una enseñanza geográfica para humanizar la sociedad tan particularizada, individualizada y consumidora.

Por tanto, ante la circunstancia contradictoria, adversa y catastrófica del mundo actual, el desafío es formar la conciencia ambiental, geográfica y social-colectiva, a partir de entender críticamente los sucesos habituales del enrevesado mundo globalizado. Se requiere alfabetizar para echar las bases del afecto hacia el ámbito planetario que habita la humanidad, de manera solidaria y responsable.

\section{Consideraciones finales}

Desde el inicio del nuevo milenio, se critica la formación educativa ante el desfase de su enrevesada realidad sociohistórica y el uso de teorías elaboradas desde la reflexión geográfica del norte, pues su aplicación se realiza en realidades lejanas a su origen. Lo debatible es la forma como se transfieren ideológica y políticamente esos conocimientos y prácticas de situaciones ajenas y disímiles a la región latinoamericana y caribeña.

Por eso inquieta preservar la geografía descriptiva como opción científica para alfabetizar geográficamente, con fundamentos de la pedagogía y la didáctica tradicional-conductista, ambas consideradas básicas para orientar la labor formativa de estudiantes que viven en países monoproductores de materias primas, y dependientes de los adelantos científicos y tecnológicos del norte industrializado.

Una razón criticable es la forma como se desvía la explicación de los contratiempos originados por el proceso colonial y las imposiciones del mundo globalizado, al enseñar geografía para impedir el análisis de la condición de países consumidores de conocimientos y prácticas impuestos desde el eurocentrismo y el nuevo orden mundial para formar los ciudadanos del sur.

En esta situación emergen tres aspectos indispensables por sus significativas implicaciones: en primer lugar, urge aprovechar los avances epistémicos de la ciencia geográfica para estudiar las adversas problemáticas y formar conciencia crítica; en segundo lugar, promover cambios pedagógicos y didácticos para formar ciudadanos que mejoren su calidad de vida colectiva; $y$, en tercer lugar, educar en valores como la responsabilidad y la solidaridad. Por eso es necesario:

1. Continuar con la denuncia razonada sobre la vigencia de la enseñanza de la geografía decimonónica y la necesaria innovación explicativa de la realidad geográfica desde su renovación disciplinar. Preocupa el carácter inalterable de la geografía precientífica en la acción educativa desarrollada en la práctica escolar cotidiana, dado su acento transmisor de contenidos programáticos obsoletos.

2. Revisar la situación de la práctica pedagógica de la geografía escolar según las remozadas orientaciones epistémicas de la pedagogía y la didáctica para ejercitar la elaboración del conocimiento. El apego al tradicionalismo decimonónico impide que los ciudadanos razonen lo complicado del mundo, la realidad y la vida en el ámbito del nuevo orden económico mundial.

3. Entender que el desafío geográfico y formativo es desarrollar los procesos de enseñanza y aprendizaje desde la investigación como labor didáctica. En efecto, implica utilizar el contenido programático desde la acción-reflexión-acción crítica para direccionar su intervención pedagógica en la explicación de la realidad geográfica vivida y educar integralmente la personalidad del educando.

4. Abordar los problemas geográficos que viven las comunidades como objetos de estudio de la geografía escolar. Se trata de asumir el entorno inmediato para educar vivencialmente al abordar los temas y las problemáticas porque afectan la geografía comunitaria. Es analizar las circunstancias del lugar desde el sentido común, la intuición y la investigación en la calle.

Por tanto, la inquietud innovadora apunta a develar los acontecimientos habituales de la práctica escolar cotidiana de la enseñanza geográfica, como la base de la gestión innovadora de la acción formativa que pretende fomentar la educación geográfica ante lo caótico y confuso del actual momento histórico. Es educar para fortalecer comportamientos humanos hacia el complicado entorno inmediato afectado por sus acciones anárquicas al usar el territorio ocupado.

El apremio obedece a lo acentuado de las complicaciones y adversidades geográficas con efectos angustiantes para los ciudadanos. Por eso, la premura de una tarea impostergable de la geografía escolar ante la complejidad del mundo contemporáneo debe ser contribuir a alfabetizar, con la acción indagadora de los acontecimientos ambientales, geográficos y sociales que afectan la calidad de vida comunitaria, a la forma anárquica de intervenir el territorio y organizar el espacio.

La construcción del conocimiento se ejercitará con la acción formativa del aula como la tarea esencial para apuntalar la enseñanza geográfica en forma pertinente con los desafíos enfrentados actualmente por América Latina y el Caribe. Eso implica fundar la enseñanza de la geografía en una orientación más humana y social, que sensibilice las colectividades sobre su condición de actores protagonistas de los cambios geohistóricos del nuevo milenio con participación crítica y constructiva. 


\section{Referencias}

Caldera Rodríguez, A. (8 de julio del 2008). Para una formación holística del ciudadano. Diario Panorama, 1-3.

Carretero, M. (2009). Constructivismo y educación. Buenos Aires: Paidós.

Claval, P. (1992). La géographie d'aujourd'hui: comment l'enseiger. L'information Géographique, 56, 71-82.

Delgado Mahecha, O. (2003). Debate sobre el espacio en la geografía contemporánea. Bogotá: Universidad Pedagógica Nacional.

Díaz Piña, J. (1996). El nuevo contexto geocultural y sus implicaciones para el Área de Estudios Sociales en la Educación Básica. Caracas: Ministerio de Educación.

Díaz, F. y Hernández, G. (2002). Estrategias docentes para un aprendizaje significativo una interpretación constructivista ( $2^{\mathrm{a}}$ ed.). México D.F.: McGraw-Hill Interamericana.

Giroux, H. (2009). Educación contextualizada. Recuperado de henry_giroux.blogspot/2009/05/educación_ contextualizada

González Velasco, J. M. (2009). Didáctica critica desde la transdisciplinariedad, la complejidad y la investigación. De cara a los retos y perspectivas educativas del devenir de nuestros tiempos. Revista Integra Educativa, II(1), 63-74.

Gurevich, R. (1994). Un desafío para la geografía: explicar el mundo. En Didáctica de las ciencias sociales (pp. 125152). Buenos Aires: Paidós.

Hernández, K. (2011). La educación en Venezuela. Caracas: Consultores Asociados.

Lacueva Teruel, A. y Manterola, C. (1989). La producción de textos escolares como una línea de trabajo en las instituciones de formación docente. Nuestra experiencia. Revista de Pedagogía, (17), 30-37.

Moreno, L. N., Rodríguez, P. L. y Sánchez, A. J. (2007). Educación geográfica, conocimiento social y formación ciudadana. En La función social de la geografía en América Latina (pp-50-75). México D.F.: Academia Española.

Pipkin, D., Varela, C. y Zenobi, V. (2001). Aportes para el debate curricular. Enseñanza de las Ciencias Sociales. Buenos Aires: Secretaría de Educación, Dirección de Currícula, Gobierno de la Ciudad Autónoma de Buenos Aires.

Rodríguez de Moreno E., A. (2000). Geografía conceptual. Bogotá: Tercer Mundo.

Rodríguez Ebrard, L. A. (2008). Vínculo entre la investigación-acción, el constructivismo y la didáctica crítica. Odiseo. Revista Electrónica de Pedagogía, 5(10). Recuperado de http://www.odiseo.com.mx/2008/5-10/rodriguez-vinculo.html 